\title{
Moses Effect (Deformation of Diamagnetic Liquid/Vapor Interface by Magnetic Field): Physics and Applications
}

\author{
Edward Bormashenko \\ Ariel University, Engineering Faculty, Chemical Engineering, Biotechnology and \\ Materials Department, P.O.B. 3, 407000, Ariel, Israel
}

Corresponding author: Edward Bormashenko

E-Mail: Edward@ariel.ac.il

Phone: 972-3-074 7296863

\begin{abstract}
Deformation of the surface of diamagnetic liquid by magnetic field is called the "Moses Effect". Physics and applications of the direct and inverse Moses effects are reviewed. Experimental techniques enabling visualization of the effects are surveyed. Impact of magnetic field on micro- and macroscopic properties of liquids is addressed. Influence of the surface tension on the shape of the near-surface dip formed in a diamagnetic liquid by magnetic field is reported. Floating of diamagnetic bodies driven by the Moses effect is treated. The effect of the "magnetic memory of water" in its relation to the Moses Effect is discussed. The dynamics of self-healing of near-surface dips due to the Moses Effect is considered.
\end{abstract}

Keywords: diamagnetic liquid surface; magnetic field; Moses Effect; interfacial tension; shape of the near-surface dip.

\section{Introduction}

The application of electrical and magnetic fields on interfaces gives rise to numerous physico-chemical events, one of the most important of which is deformation of the liquid/vapor interface [1-10]. The phenomena arising from application of electric field to the liquid/vapor interface were studied by outstanding scientists, such as Rayleigh [1], Chandrasekhar and Fermi [3] and Taylor [6-7]. One of the most important effects arising from the application of the electric field on the liquid/vapor interface is the formation of the so called Taylor cone [5-8]. When a liquid is exposed to an electric field, the shape of liquid starts to deform from the shape caused by surface tension alone. As the voltage is increased the effect of the electric field becomes more prominent. As this effect of the electric field begins to exert a similar magnitude of force on the droplet as the surface tension does, a cone shape begins to form with convex sides and a rounded tip. When a threshold voltage has been 
reached the slightly rounded tip inverts and emits a jet of liquid [10-11]. When threshold voltage is applied to polymer solution it jets a fiber, thus giving rise to the electro-spinning process, enabling industrial manufacturing of polymer fibers with a diameter of some hundred nanometers $[7,11]$.

Quite surprisingly an impact of the magnetic field on the liquid/vapor interface (known also as the "Moses Effect") is less studied and understood. When a field of $c a 0.5-10 \mathrm{~T}$ is applied on the surface of water it is macroscopically lowered $\left(h \cong 10-10^{4} \mu \mathrm{m}\right)$ at the field center, relative to the level at the zero field region, as shown in Figures 1, 2A.

Regrettably three very different physical phenomena are regarded in parallel in the literature as the "Moses Effects". Extremal water repellant properties of the surfaces decorated with the arrays of glass cone spikes hydrophobized with a fluorinated silane solution get the title of the "Moses" effect in Refs. 12-13. The scaling law related to physics of stochastic processes evolving over a time is also called "the Moses effect" [14]. The stochastic processes in which time scaling exponent are anomalous is referred to as the Moses effect and characterized by the Moses exponent $M$, which quantifies the growth of the increment distribution [14]. Chen et al. labeled the phenomenon the Moses effect due to the Bible story describing how Moses led the Israelites after their Exodus from Egypt as they wandered through the wilderness having no stationary settlements [14].

In the presented paper we address the effect of deformation of the liquid/vapor interface by the magnetic field also called the Moses Effect [15-17]. According to the Exodus account, Moses held out his staff, and the Red Sea was parted by God, thus in our case the magnetic field serves as Moses staff, dividing water. The Moses effect is an essentially macroscopic one. However, we start our review from the microscopic aspect of influence exerted by magnetic field on water.

\section{Impact of magnetic field on water: the microscopic approach.}

In spite of the enormous experimental effort spent for the study of effect of the magnetic field on the properties of water, the results reported in the field remain controversial [18]. One of the most obscure phenomena is the effect of the "magnetic memory of water", namely, the long term effect, which presumably persist for hours or days after water treatment with the electromagnetic and/or magnetic fields. Some of researchers try to explain the effect "magnetic memory of water" as an experimental error or something which is not physically possible [18]. It has been reported that preliminary water treatment with magnetic or electromagnetic (EM) fields can help descale metal surfaces, improve cement hydration, 
change $\zeta$ potential of colloids, make plants irrigated with such water grow faster, enhance efflux of calcium through biomembranes or influence the structure of model lyposomes. The effects persist minutes or hours after the water treatment. It is well known that relaxation phenomena in water occur on a picosecond to second timescale. The nature of these obscure and questionable phenomena uniquely known as the 'magnetic memory of water' has recently been scrutinized. It was suggested that the vapor/liquid interface is perturbed by the action of magnetic and electromagnetic fields (in our wording by the Moses effect). The magnetic treatment of water gives rise to formation free radicals and/or reactive oxygen species are observed after the treatment (ozone, superoxide, hydroxyl radicals, singlet oxygen, atomic hydrogen, hydrogen peroxide, etc.). The perturbations of the vapor/liquid interface according to ref. 18 relax more slowly (minutes to hours). The presence of gases, such as carbon dioxide or noble gases which promote clathrate-like structures of water, significantly enhanced the observed effects. It was speculated that the effect of the "magnetic memory of water" is the combination of perturbations of the vapor/ liquid interface and the production of reactive oxygen species [18]. Thus, it was suggested that the effect of the "magnetic memory of water" is at least partially due to the Moses Effect [18].

It was also reported in ref. 19 that a magnetic field exists for carbonates formed by heating water exposed to static magnetic field of $c a \cong 0.1 T$. Passing water through a magnetic field subsequently favors formation of aragonite rather than calcite in the experiments reported in ref. 19, and the influence of the treatment persists for more than two hundred hours. It was also communicated that water molecules tend to form clusters via hydrogen bonds under magnetic treatment [20]. The additional experimental and theoretical efforts definitely are necessary for the clarification of the problem of the "magnetic memory of water" [18]. The macroscopic aspect of the problem will be considered below.

\section{Impact of the magnetic field on the macroscopic properties of water.}

Ref. 24 addressed the effect of the magnetic field on macroscopic properties of water. Purified water was circulated at a constant flow rate in a magnetic field. After this treatment, the properties of water were changed, demonstrating the decrease of surface tension and the increase of viscosity over the treatment time [20]. On contrast, an increase of the surface tension of water exposed to magnetic field was reported in ref. 21. The effect of the change in surface tension is weak, thus more experiments are definitely desirable. It was also shown recently that magnetic field changed physical properties of water, including specific heat, evaporation amount and the boiling point [22]. It was also reported that evaporation rate of 
water increased markedly after magnetic field treatment [22]. It makes sense to compare the surprising results demonstrated in Ref. 22 with those reported in ref. 23, in which it was shown that evaporation rate of water increased under electric field treatment. It was found that the electric field in the direction perpendicular to the water surface enhances the water evaporation, while the electric field in the direction parallel to the water surface impedes the water evaporation [23]. This finding qualitatively agrees with the experimental observation related to electrospinning $[7,11]$ and is reasonably explained in terms of the water dipole alignment by the electric field [23]. The available today possibility to create strong magnetic fields with permanent magnets $B \approx 1-10 T$ calls for further experimental investigations which will shed light on the influence of magnetic fields on the magnetic properties of water.

\section{The statics of the Moses effect.}

Consider a steady magnetic field (produced say by a Neodymium magnet) applied to the diamagnetic liquid/vapor interface as depicted in Figures 1, 2A. Diamagnetic bodies as it is well known are repelled by steady magnetic fields [15]. Diamagnetism is usually caused by small distortion of the electron orbits within atoms or molecules by the applied magnetic field. Magnetic dipoles are induced that oppose the external field [15]. Macroscopically this results in the formation of the "well" created in the liquid, and depicted in Figure 1. Let us consider the case of an axisymmetric magnetic field that depends only on the radius (in a horizontal direction): $\mathbf{B}=\mathbf{B}(r)$. Dependence of the magnetic field on the depth $B(\mathrm{z})$ is neglected. We assume that the well shape is also axisymmetric, with the surface described as $z=-h(r)$, with $h(r) \rightarrow 0, r \rightarrow \infty$ and $h(r)$ bounded for all $r$. These conditions imply that the characteristic size of the vessel is assumed to be much larger than the characteristic scale for decay of the magnetic field. Then, to find the shape of the well, we consider the energy balance caused by application of the field. There are three contributions to the energy balance [24]:

- Energy gained due to driving the liquid out of the field due to the diamagnetic force (denoted as $\Delta E_{\mathrm{mag}}$ );

- Energy loss due to elevation of the liquid in the vessel (denoted as $\Delta E_{\mathrm{gr}}$ );

- Energy loss due to extension of the liquid surface (denoted as $\Delta E_{\text {surf }}$ );

The detailed analysis of the problem of the establishment of exact shape of the well is supplied in ref.

$$
h(r)=\frac{\chi \mathbf{B}^{2}(r)}{2 \rho g \mu_{0}}
$$


Equation 1 is a well-known expression, supplied explicitly in the seminal Ref. 15. It should be emphasized that $h(r)$ scales as $B^{2}$; thus, switching the direction of the magnetic field will not influence the shape of the near-surface dip, shown in Figure 1. Consider also that eq. 1 does not contain the surface tension of a liquid, deformed by magnetic field due to the latently adopted suggestion that the capillary length is small compared to typical scale of variation of the magnetic field [24]. Actually, the shape of the well is substantially affected by the surface tension of the liquid, and therefore a more accurate expression of eq. 1 should be used, as derived in Ref. 24. The exact expression, describing the shape of the well is very complicated, and may be established only numerically [24]. However, the experiments carried out with a variety of liquids (water, silicon oil and surfactant solutions) demonstrated that the effect of the magnetic field $B \cong 0.35 T$ ) is not negligible [24].

Various experimental techniques were reported, enabling static visualization of the Moses Effect. Witty technique, allowing naked eye observation of the Moses Efect using the digital single-lens reflex (DSLR) camera is reported in ref. 25. Usually, visualization of the deformation of liquid surfaces, deformed by magnetic fields involves the laser illumination of the surfaces followed by the analyzing the reflection patterns from the curved liquid surface [24, 26-27]. The accuracy of the methods involving the laser illumination for the establishment of the precise shape of the near-surface wells due to the Moses Effect is $c a 0.5$ $\mu \mathrm{m}$ and it is expectably close to the wavelength of the laser exploited for the illumination [24].

\section{The dynamics of the Moses effect; self-healing of the dip created by a magnetic} field.

Consider characteristic spatial and temporal times of self-healing the well created by the magnetic field at the liquid/vapor interface (say when the applied magnetic field falls to zero). There exist two main pathways of the self-healing of the well, shown in Figure 1, namely: inertial and viscous, governed by inertial and viscous forces correspondingly [2831]. Let us compare the contributions of the inertial and viscous forces. The process of coalescence is driven by capillarity tending to diminish the surface of a liquid, the viscosity $\eta$ tends to withstand the coalescence [28-31]. Thus, the characteristic velocity $v_{c a}$ built from the main physical characteristics of the process should be introduced:

$$
v_{c a}=\frac{\gamma}{\eta}
$$


where $\gamma$ and $\eta$ are the surface tension and viscosity of the liquid correspondingly. The characteristic velocity $v_{c a}$ is called the capillary velocity [28-31]. Now we compare the impact of viscous and inertial forces with the use of the Reynolds number [28-29], assuming $v \cong v_{c a}:$

$$
R e=\frac{\rho v_{c a} h}{\eta}=\frac{\rho \gamma h}{\eta^{2}}
$$

where $\rho$ is the density of a liquid; in turn $h \cong 10-10^{4} \mu m$ is the experimentally established depth of the well (see Figure 1) and the capillarity velocity $v_{c a}$ (given by Eq. 2) are assumed as the characteristic dimension and velocity, correspondingly (consider that the lateral dimension of the "well" (namely $R$, shown in Figure 1) is governed be the lateral dimension of the Neodymium magnet, usually used in the investigation). It is reasonable to anticipate, that viscous coalescence occurs for $R e<1$, and inertial coalescence takes place for $R e>1$. If we assume that the crossover between the two regimes happens at $\operatorname{Re} \sim 1$ (as it was verified experimentally in Ref. 28, this yields a characteristic length and time scale (denoted $R_{\text {in }}$ and $\tau_{\text {in }}$ ) correspondingly beyond which inertia should become important;

$$
\begin{gathered}
R_{i n} \approx \frac{\eta^{2}}{\rho \gamma}, \\
\tau_{i n} \approx \frac{\eta^{3}}{\rho \gamma^{2}} .
\end{gathered}
$$

Substituting into Eqs. 3-4 the corresponding values for water, namely $\eta \cong 9 \times 10^{-4} \mathrm{~Pa} \times$ $s ; \rho \cong 10^{3} \frac{\mathrm{kg}}{\mathrm{m}^{-3}} ; \gamma \cong 70 \times 10^{-3} \frac{\mathrm{N}}{\mathrm{m}}$ at ambient conditions, we find $R_{\text {in }} \approx 15 \mathrm{~nm}, \tau_{\text {in }} \approx 10^{-10} \mathrm{~s}$. Obviously, for water there is no hope of observing the viscous regime of self-healing of nearsurface wells, created by magnetic field, and the process of self-healing of these wells is from a practical point of view always inertial [28, 31]. Thus, the idea that the deformation of surface inspired by magnetic fields is responsible for the long lasting effect of "water memory" seems to be unrealistic.

On the other hand for high viscous silicon oils we obtain from eqs. 4-5 rather different values of the characteristic spatial and temporal scales; namely substitution of $\eta \cong$ $1.0 P a \times s ; \rho \cong 900 \frac{\mathrm{kg}}{\mathrm{m}^{3}} ; \gamma \cong 20 \times 10^{-3} \frac{\mathrm{N}}{\mathrm{m}}$ into eqs. 2 , 4-5 yields $v_{c a} \cong 20 \frac{\mathrm{mm}}{\mathrm{s}} ; R_{\text {in }} \cong$ $1.5 \mathrm{~m} ; \tau_{\text {in }} \cong 3 \mathrm{~s}$. Thus, the regime of self-healing of millimetrically scaled dips is viscous. 
It is reasonable to assume that the characteristic time of viscous self-healing scales as $\tau_{v i s c} \cong$ $\frac{R}{v_{c a}}$ (see Figure 1). Adopting $R \cong 10 \mathrm{~mm}$ yields $\tau_{v i s c} \cong 0.5 \mathrm{~s}$. Hence, we conclude that for the viscous liquids (such as highly viscous silicon oils) the effect of "magnetic memory" is observable on the "large" spatial and temporal scales, inherent for viscous self-healing of near-surface wells due to the Moses effect.

\section{Physical phenomena arising from the Moses effect.}

The Moses effect was addressed experimentally in ref. 17. The quantitative experimental research focused on magnetic field effects on the surface profile have been reported for water and copper sulfate aqueous solutions that have very small magnetic susceptibility. When a magnetic field of $c a 10 \mathrm{~T}$ was applied on a horizontal superconducting magnet, the surface of distilled water was lowered by $39 \mathrm{~mm}$ at the field center, relative to the level at the zero field region. In contrast, the surface of a paramagnetic nearly saturated copper sulfate aqueous solution was raised by roughly the same height at the center. This effect was labeled in Ref. 17 as the "reversed (inverse) Moses effect", shown in Figure 2B. The obtained profiles were interpreted with eq. 1 involving the dia- and paramagnetic volume susceptibility values of distilled water and copper sulfate aqueous solution, respectively. Ref. 32 addressed the change in the interface profile between immiscible nonmagnetic liquids. The interface profile changed into concave down or up at the field center accordingly to the balance of magnetic susceptibilities between the lower and upper liquids [32]. The interpretation of the experimental results was based on eq. 1 neglecting the impact of the interfacial tension [24]. A flat interface was also demonstrated when the susceptibilities were balanced [24]. It was found that modification of the interface profile was significantly amplified under an applied magnetic field as low as $1 \mathrm{~T}$ when the densities of the two liquids were close. It was suggested that the interfacial change induced by the applied magnetic field can be used to remove a boundary, which initially separates two liquids without the field, and to initiate a mixing process or a chemical reaction between the two liquids [32]. Selfassembly of nonmagnetic gold spheres immersed in a 40 mass $\% \mathrm{MnCl}_{2}$ solution was addressed in ref. 33. The authors related the effect of self-assembly to interactions between magnetic dipoles induced in the nonmagnetic spheres and magnetic force derived from field gradient [33]. The capillary (interfacial) interactions between gold spheres, thus, were neglected [33].

Periodic magnetic field was successfully used to trap polystyrene spheres $(20 \mu \mathrm{m}$ in diameter suspended in a liquid) in a periodic line pattern [34]. Upon drying, the trapped 
spheres formed the self-organized packing [34]. Ferromagnetic bumps effectively dewetted using the micro-Moses effect, resulting in formation of contact holes on the bumps were reported in Ref. 35. This idea gave rise to an electrical contact complementary to a flip-chip structure [35]. Magneto-Archimedes levitation in a strong fields, based on the repulsive diamagnetic force from the magnetic field, balancing with the weight of the substance was discussed in Ref. 16.

It was demonstrated recently that diamagnetic objects (polymer and metallic plates and spheres, ceramic beads, and liquid marbles), floating on water, and a variety of organic diamagnetic liquids (namely: $\left(\mathrm{H}_{2} \mathrm{O}\right)$, Ethanol $\left(\mathrm{C}_{2} \mathrm{H}_{5} \mathrm{OH}\right)$, Diiodomethane $\left(\mathrm{CH}_{2} \mathrm{I}_{2}\right)$ and Dichloromethane $\mathrm{CH}_{2} \mathrm{Cl}_{2}$ )) may be driven by a steady magnetic field as low as $0.1 \mathrm{~T}$, as registered at the liquid-vapor surface [36]. Diamagnetic bodies were attracted to the magnet (as shown in Figure 3), when the apparent contact angle as the solid/liquid interface is obtuse and repelled from the magnet, when the angle was acute. Cold plasma-treated polyolefin rafts and spheres, demonstrating underwater floating, were repelled by a permanent magnet [36]. Addition of a surfactant to the water, as well as cold plasma treatment of the polyolefin bodies, switched the attraction into the repulsion. We conjectured that the observed effects were caused by the interplay of two main phenomena [36]. The first of which is the gravity, which induces sliding of the particle on the deformed liquid/vapor interface, created by the Moses Effect. The second cause is the hysteresis of the contact angle at the bodies' boundary [37-42]. The interrelation between gravity and interfacial phenomena is addressed in ref. 24 .

The role of the diamagnetic repulsion in the motion of floating diamagnetic bodies was experimentally treated in refs. 24, 36. It was demonstrated that driving millimetrically scaled, soap and glycerol bubbles floating on a liquid, using a steady magnetic field of $c a 0.5 \mathrm{~T}$, is possible [43]. The experiments with bubbles demonstrated that the role of bulk forces such as gravity and diamagnetic repulsion in the displacement of floating diamagnetic objects driven by steady magnetic fields may be negligible [43]. The mathematical model of the process was suggested [24, 43].

The use of paramagnetic $\mathrm{HoCl}_{3}$ solutions for controlled magnetically driven displacement of droplets and liquid marbles [38, 9, 44-51] exploiting the "inverse Moses effect" [25] was reported recently [52]. It should be emphasized that exploiting "the Moses effect" and the inverse (reverse) Moses effect enables excluding ferromagnetic particles, usually used for magnetic manipulation of liquids [53-61]. Manipulation of ferromagnetic, paramagnetic and entities with magnetic fields was reviewed in ref. 62. Magnetically driven 
movement of biological fluid droplets placed on non-patterned, silicon nanowire superhydrophobic surfaces was communicated in ref. 63. Magnetic control over liquid surface properties attained with responsive magnetic surfactants was reported in ref. 64 . It was demonstrated that liquid emulsions containing magneto-responsive surfactants may be controlled by application of an external magnetic field [64]. Interesting interfacial effect driven by the magnetic-field gradient is reported in ref. 65. The magnetic-field gradient gave rise to the Moses effect and enabled the insertion and confinement of hydrophobic metallic powders in water, based on encapsulating an air bubble surrounded by a hydrophobic metallic shell similar to those inherent to liquid marbles [9, 44-51, 65]. Interface profile evolution between binary immiscible fluids induced by high magnetic field gradients was reported in ref. 66 (see also ref. 32). Approaches to magnetic treatment of water were surveyed recently in ref. 67. Magnetic properties of diamagnetic liquids are summarized in Ref. 68.

\section{Conclusions}

Application of magnetic field on liquids leads to numerous bulk and surface phenomena [1-8], one of which is the deformation of the diamagnetic liquid/vapor interface by magnetic field, called also as the "Moses Effect" [16-17, 32]. The rapid progress in the development of Neodymium magnets, supplying magnetic fields as high as ca 1T, allows simple and inexpensive experiments related to the Moses effect and its visualization [25-27]. Applications of the magnetic fields at the order of magnitude of 0.5-1 $\mathrm{T}$ results if the formation of the near-surface "well" with a depth of dozens of micrometers. Application of magnetic field to paramagnetic liquids creates liquid bulges, and it is called the "inverse Moses Effect" [16, 25]. It is usually latently suggested that the shape of the well arises from the interplay of magnetic force and gravity. Recent experiments performed with organic liquids and solutions of surfactants demonstrated that the surface tension of liquids is also essential for the formation of near-surface dips, resulting from the application of magnetic fields [24]. Impact of the magnetic field on the macroscopic properties of liquids (surface tension and evaporation rate) is reviewed. Dynamics of self-healing of the surface deformations due to the Moses effect is addressed. Application of magnetic fields $(B \cong$ $0.5 T$ ) on diamagnetic liquid/vapor interfaces enables driving of floating diamagnetic bodies and soap bubbles [36, 43].

\section{Acknowledgements}

The author is indebted to Dr. M. Frenkel and Mrs. Ye. Bormashenko for their kind help in preparing this manuscript. 


\section{References}

1. Rayleigh, L. On the equilibrium of liquid conducting masses charged with electricity. Philos. Mag. 1882, 14 (87), 184-186.

2. Tonks, L. A Theory of liquid surface rupture by a uniform electric field. Phys. Rev. 1935, 48, 562-568.

3. Chandrasekhar, S.; Fermi, E. Problems of gravitational stability in the presence of a magnetic field. Astrophys. J. 1953, 118, 113-141.

4. Nayyar, N. K.; Murty, G. S. The flattening of dielectric liquid drop in a uniform electric field. Proc. Natl. Inst. Sci. India, Part A 1959, 25 (6), 373-379.

5. Zeleny, J. Instability of electrified liquid surfaces. Phys Rev. 1917, 10 (1), 1-6.

6. Wilson, C. T. R.; Taylor, G. I. The bursting of soap-bubbles in a uniform electric field. Math. Proc. Camb. Philos. Soc. 1925, 22 (5), 728-730.

7. Taylor, G. I. Disintegration of water drops in an electric field. Proc. R. Soc. Lond. A 1964, 280 (1382), 383-397.

8. Yarin, A. L. Taylor cone and jetting from liquid droplets in electrospinning of nanofibers. J. Appl. Phys. 2001, 90, 4836-4846.

9. Bormashenko, Ed.; Pogreb, R.; Balter, R.; Gendelman, O.; Aurbach, D. Composite non-stick droplets and their actuation with electric field. Appl. Phys. Lett. 2012, 100, 151601.

10. Joffre, G.; Prunet-Foch, B.; Berthomme, S.; Cloupeau, M. Deformation of liquid menisci under the action of an electric field. J. Electrostatics, 1982, 13 (2), 151-165.

11. Wendorff, J.-H.; Agarwal, S.; Greiner, A. Electrospinning: Materials, Processing, and Applications, Wiley-VCH, Weinheim, Germany

12. D’Urso, D., Simpson, J. T., Kalyanaraman, M. Emergence of superhydrophobic behavior on vertically aligned nanocone arrays. Appl. Phys. Lett. 2007, 90, 044102.

13. Simpson, J. T., Hunter, S. R. Aytug, T. Superhydrophobic materials and coatings: a review. Rep. Prog. Phys. 2015, 78, 086501. 
14. Chen, L.; Bassler, K. E.; McCauley, J. L.; Gunaratne, G. H. Anomalous scaling of stochastic processes and the Moses effect. Phys. Rev. E. 2017, 95, 042141.

15. Landau L.; Lifshitz, E. Electrodynamics of continuous media, Vol. 8 of the Course of Theoretical Physics, Pergamon, Oxford, 1960.

16. Kitazawa, K.; Ikezoe, Y.; Uetake, H.; Hirota, N. Magnetic field effects on water, air and powders. Physica B 2001, 294-295, 709-714.

17. Hirota, N.; Homma, T.; Sugawara, H.; Kitazawa, K.; Iwasaka, M.; Ueno, Sh.; Yokoi, H.; Kakudate, Y.; Fujiwara, Sh.; Kawamura, M. Rise and fall of surface level of water solutions under high magnetic field. Jpn. J. Appl. Phys. 1995, 34, L991.

18. Colic, M.; Morse D. The elusive mechanism of the magnetic 'memory' of water, Colloids \& Surfaces A 1999, 154, 167-174.

19. Coey, J. M. D.; Cass, St. Magnetic water treatment. J. Magnetism \& Magnetic Materials 2000209 (1-3), 71-74.

20. Cai, R.; Yang, H.; He, J.; Zhu, W. The effects of magnetic fields on water molecular hydrogen bonds. J. Molecular Structure, 2009, 938, 15-19.

21. Fujimura, Y.; Iino, M. Magnetic field increases the surface tension of water. $J$. Physics: Conference Series 2009, 156, 012028

22. Wang, Y.; We, H.; Li, Z. Effect of magnetic field on the physical properties of water. Results in Physics 2018, 8, 262-267.

23. Okuno, Y.; Minagawa, M.; Matsumoto, H.; Tanioka, A. Simulation study on the influence of an electric field on water evaporation. J. Molecular Structure: THEOCHEM 2009, 904, 83-90.

24. Gendelman, O.; Frenkel. M.; Fliagin V.; Ivanova N. I.; Danchuk V.; Legchenkova, I.; Bormashenko, Ed. Study of the displacement of floating diamagnetic bodies by magnetic field. 2019, Submitted to Surface Innovations.

25. Laumann, D. Even liquids are magnetic: observation of the Moses effect and the inverse Moses Effect. Phys. Teach. 2018, 56, 352-354.

26. Chen, Z.; Dahlberg, E. D. Deformation of water by a magnetic field. Phys. Teach. 2011, $49,144-146$.

27. Dong, J.; Miao, R.; Qi, J. Visualization of the curved liquid surface by means of the optical method. J. Appl. Physics 2006, 100, 124914.

28. Eggers J.; Lister J. R.; Stone H. A. Coalescence of liquid drops. J. Fluid Mech. 1999, 401, 293-310. 
29. Aarts, D. G. A. L.; Lekkerkerker, H. N. W.; Guo, H.; Wegdam, G. H.; Bonn, D. Hydrodynamics of droplet coalescence. Phys. Rev. Lett. 2005, 95, 164503.

30. Duchemin, L.; Eggers, J.; Josserand, C. Inviscid coalescence of drops. J. Fluid Mech. 2003, 487, 167-178.

31. Bormashenko, Ed. Physics of Wetting. Phenomena and Applications of Fluids on Surfaces, de Gruyter, Berlin, Germany, 2017.

32. Sugawara, H; Hirota, N.; Homma, T. et al. Magnetic field effect on interface profile between immiscible nonmagnetic liquids - Enhanced Moses effect. J. Appl. Physics 1996, 79, 4721 .

33. Takayama, T.; Ikezoe, Y., Uetake, Y. et al. Self-organization of nonmagnetic spheres by magnetic field. Appl. Phys. Lett. 2005, 86, 234103.

34. Kimura, T.; Yamato, M.; Nara, A. Particle trapping and undulation of a liquid Surface using a microscopically modulated magnetic field. Langmuir 2004, 20 (3), 572 - 574.

35. Uemura T.; Kimura, T.; Sugitani, M; Kumakura, M. Formation of contact holes on bumps on semiconductor chip by Micro-Moses Effect. Adv. Mat. 2006, 18 (12), 15491551.

36. Frenkel, M., Danchuk, V.; Multanen, V.; Legchenkova, I.; Bormashenko, Ye.; Gendelman, O.; Bormashenko Ed. Toward an understanding of magnetic displacement of floating diamagnetic bodies, I: Experimental findings. Langmuir 2018, 34 (22), $6388-6395$.

37. Starov, V. Equilibrium and Hysteresis Contact angles. Adv. Colloid \& Interface Sci. 1992, 39, 147-173.

38. de Gennes P. G., Brochard-Wyart F., Quéré D. Capillarity and Wetting Phenomena; Springer, Berlin, 2003.

39. Tadmor, R., Yadav, P. S. As-placed contact angles for sessile droplets. J. Colloid \& Interface Sci. 2008, 317, 241-246.

40. Tadmor, R. Approaches in wetting phenomena. Soft Matter 2011, 7, 1577-1578.

41. Erbil, H. Y. The debate on the dependence of apparent contact angles on drop contact area or three-phase contact line: A review. Surf. Sci. Rep. 2014, 69 (4), 325-365.

42. Hejazi, B.; Nosonovsky, M.Contact angle hysteresis in multiphase systems. Colloid Polym. Sci. 2013, 291, 329-338.

43. Legchenkova, I.; Chaniel, G.; Frenkel, M.; Bormashenko, Ye.; Shoval, Sh.; Bormashenko, Ed. Magnetically inspired deformation of the liquid/vapor interface drives soap bubbles. Surface Innov. 2018, 6 (4-5), 231-236. 
44. Aussillous, P.; Quéré, D. Liquid Marbles. Nature 2001, 411, 924- 927.

45. Bormashenko, E. Liquid Marbles, Elastic Nonstick Droplets: From Mini-Reactors to Self-Propulsion. Langmuir 2017, 33, 663- 669.

46. Nguyen, N-Tr.; Hejazian, M.; Ooi, Vh. H.; Kashaninejad, N. Recent Advances and Future Perspectives on Microfluidic Liquid Handling. Micromachines 2017, 8, 186.

47. Nguyen, N-Tr.; Ooi, Ch. H. Manipulation of liquid marbles. Microfluid. \& Nanofluid. 2015, 19 (3), 483-495.

48. Li, X.; Wang, R.; Shi, H. Song, B. Effective surface tension of liquid marbles using controllable nanoparticle monolayers. Appl. Phys. Lett. 2018, 113, 101602.

49. Li, X.; Wang, R.; Huang, Sh., Wang, Y.; Shi, H. A capillary rise method for studying the effective surface tension of monolayer nanoparticle-covered liquid marbles. Soft Matter 2018, 14, 9877-9884.

50. Tyowua, A.; Mooney J. M, Binks B. P. Janus liquid marbles containing both oil and water stabilised by silica or sericite particles. Colloids and Surfaces A 2019, 560, 288297.

51. Dupin, D. Armes, S.P.; Fujii, S. Stimulus-responsive liquid marbles. J. Am. Chem. Soc. 2009, 131 (15), 5386-5387.

52. Vialetto, J.; Hayakawa, M.; Kavokine, N.; Takinoue, M.; Varanakkottu, S. N.; Rudiuk, S.; Anyfantakis, M.; Morel, M.; Baigl, D. Magnetic actuation of discrete liquid entities with a deformable paramagnetic liquid substrate. Angew. Chem. Int. Ed. 2017, 56, 16678.

53. Nguyen, N.-T. Micro-Magnetofluidics: Interactions between magnetism and fluid flow on the microscale. Microfluid. \& Nanofluid. 2012, 12, 1- 16,

54. Zhang, Y.; Nguyen, N.-T. Magnetic digital microfluidics - a review. Lab Chip 2017, 17, $994-1008$.

55. Calcagnile, P.; Fragouli, D.; Bayer, I. S.; Anyfantis, G. C.; Martiradonna, L.; Cozzoli, P. D.; Cingolani, R.; Athanassiou, A. Magnetically driven floating foams for the removal of oil contaminants from water. ACS Nano 2012, 6 (6), 5413-5419.

56. Lin, J.; Zhou, W.; Kumbhar, A.; Wiemann, J.; Fang J.; Carpenter, E.; O'Connor, C. J. Gold-coated Iron (Fe@Au) nanoparticles: Synthesis, characterization, and magnetic field-induced self-assembly. J. Solid State Chem. 2001, 159, 26-31.

57. Doyll, P. S.; Bibette, J.; Bancaud, A.; Viovy J.-L. Self-assembled magnetic matrices for DNA separation chips. Science 2002, 295, 2237-2238. 
58. Whitesides, G. M.; Grzybowski B. Self-assembly at all scales. Science 2002, 295, 2418-2421.

59. Vandewalle, N.; Obara, N.; Lumay, G. Mesoscale structures from magnetocapillary self-assembly. Europ. Phys. J. E 2013, 36 (10), 127.

60. Lumay, G.; Obara, N.; Weyer, F.; Vandewalle, B. Self-assembled magnetocapillary swimmers. Soft Matter 2013, 9, 2420-2425.

61. den Dulk, R. C.; Schmidt, K. A.; Sabatté, G.; Liébana, S.; Prins, M. W. J. Magnetocapillary valve for integrated purification and enrichment of nucleic acids and proteins. Lab Chip 2013, 13, 106-118.

62. Rikken, R. S. M.; Nolte, R. J. M; Maan, J. C.; Hest, J. C.M.; Wilson D. A.; and Peter C. M. Christianen, P.C. M. Manipulation of micro- and nanostructure motion with magnetic fields. Soft Matter 2014, 10, 1295-1308.

63. Garcia, A. A.; Egatz-Gomez, A.; Lindsay, S. A. et al. Magnetic movement of biological fluid droplets. J. Magnetism \& Magnetic Mater. 2007, 311, 238-243.

64. Brown, B.; Bushmelev, A.; Butts, C. P.; Cheng, J.; Eastoe, J.; Grillo, I.; Heenan, R. K.; Schmidt, A. M. Magnetic Control over liquid surface properties with responsive surfactants. Angew. Chem. Int. Ed. 2012, 51, $2414-2416$.

65. Meir, Y.; Jerby, E. Insertion and confinement of hydrophobic metallic powder in water: The bubble-marble effect. Phys. Rev. E 2014, 90, 030301(R).

66. Zhao, L-J., You, Y.; Tian, Y-H.; Yang H-K.; Wang, Q.; He J-Ch. Interface profile evolution between binary immiscible fluids induced by high magnetic field gradients. Science China 2010, 53 (7) 1319-1324.

67. Chibowski, E.; Szcześ, A. Magnetic water treatment-A review of the latest approaches. Chemosphere 2018, 203, 54-67.

68. Handbook of Chemistry \& Physics, ed. By W. M. Haynes, Chapter 3, 549-553, $91^{\text {st }}$ ed. CRC Press, Taylor \& Francis, Boca Raton, FL. YSA, 2010. 


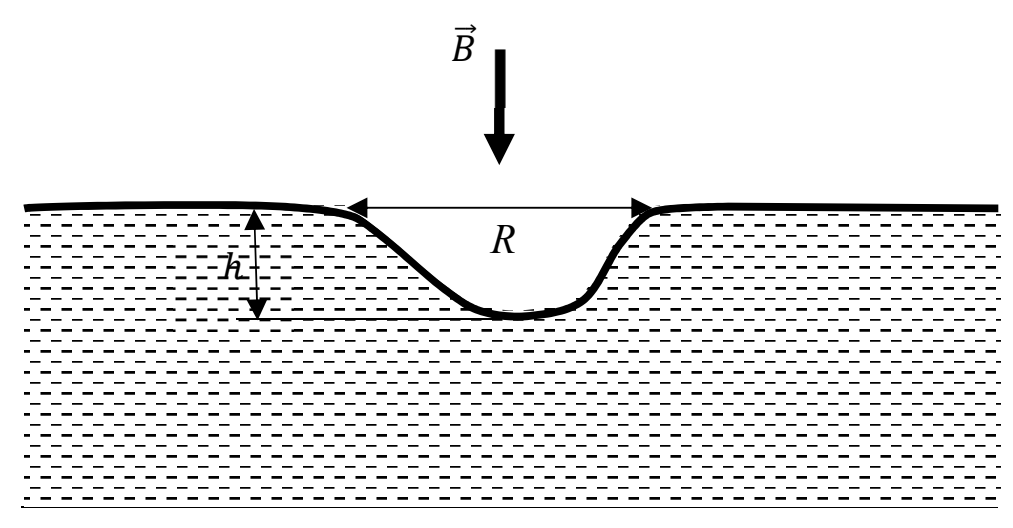

Figure 1. The Moses Effect is shown schematically. Magnetic field $\vec{B}$ creates the nearsurface dip with the depth of $h$; the lateral dimension $R$ is governed by the lateral characteristic dimension of the magnet. 


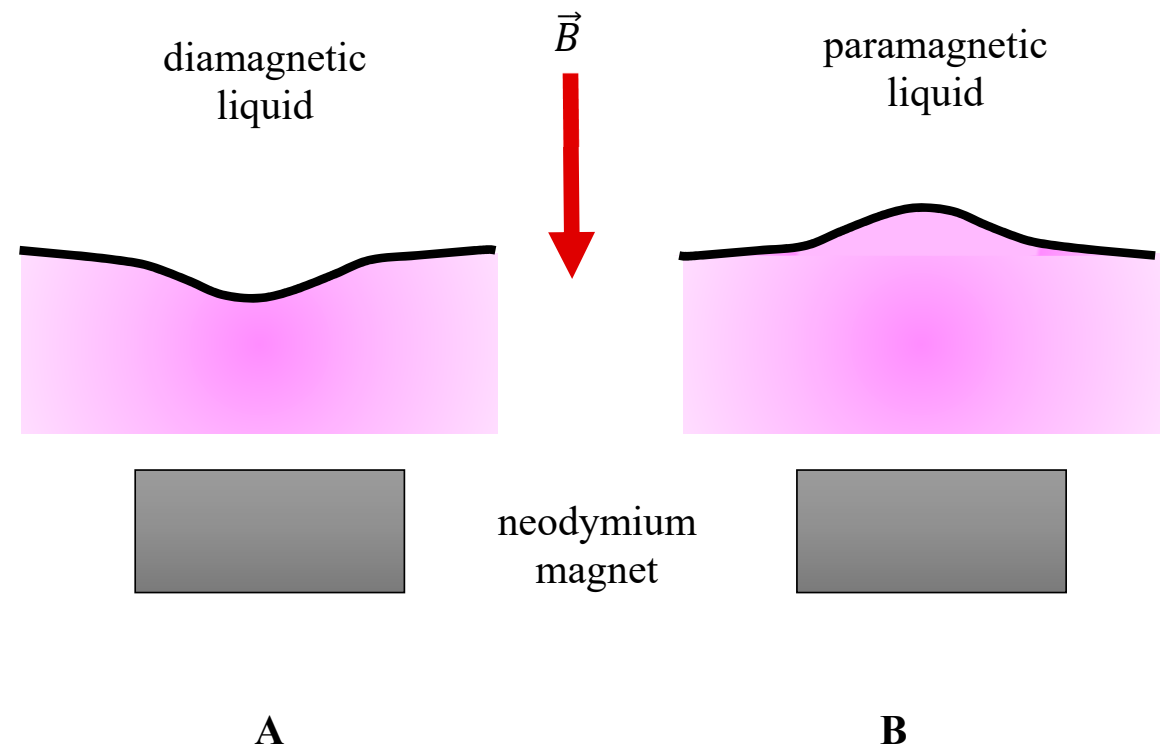

Figure 2. Direct (A) and reverse (inverse) (B) Moses Effects are depicted. 


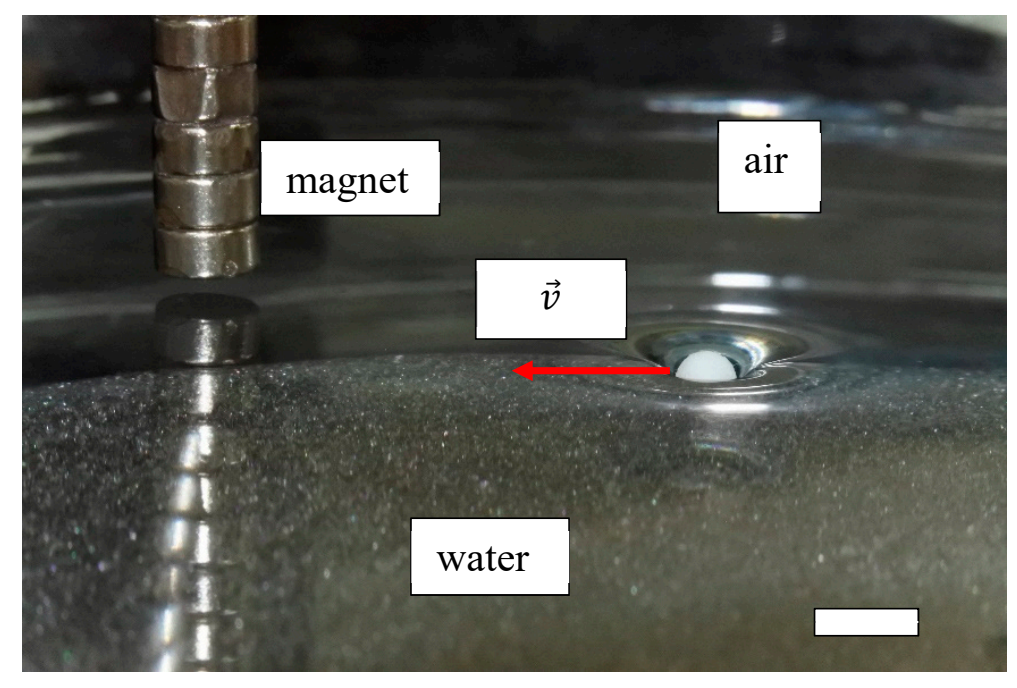

Figure 3. Floating ceramic diamagnetic ball attracted to the permanent magnet is depicted (see ref. 36). The scale bar is $5 \mathrm{~mm}$. $\vec{v}$ is the velocity of the center of mass of the ball. 